\title{
The future of medical education
}

A/Prof Yeoh Khay-Guan

$\mathrm{T}$ he medical students whom we teach today will become the doctors of tomorrow, carrying our values, skills and our hopes for the profession into the future. Therefore, it is no exaggeration to say that medical education represents the future of medicine. We have a very wide audience today, from medical students to general practitioners and specialists, so I will start with a survey of the medical education landscape today, then discuss some trends that will affect the future and how we should prepare doctors for those future needs - preparing future-ready doctors - which will be the main topic today.

\section{CURRENT LANDSCAPE}

We have three medical schools in Singapore today. Collectively, we produce 450-500 doctors each year. Other doctors return from overseas education to join the clinical service in Singapore every year. In today's talk, I will take the liberty of using examples from Yong Loo Lin School of Medicine, National University of Singapore (NUS Medicine), as I am familiar with these. Many in the audience today are associated with NUS Medicine as alumni, staff or students. For those who have graduated many years ago, I assure you that the education has changed quite a lot. For instance, our class size in NUS Medicine is 300, which is the single largest class of medical students in Singapore.

With the advent of two other schools, we had to ask ourselves: 'What is our identity?' We are proudly Singaporean in the curriculum, the teachers and the content that we teach. We have been the main medical school for most of the history of Singapore, with over 10,000 students and alumni, and a reputation that is internationally recognised. I show all students a slide about our public mission on their first day in medical school. When we were founded in 1905, it was not by the colonial government but by a people's movement led by Mr Tan Jiak Kim, who petitioned the government to set up a medical school so that Singapore would have its own doctors to serve the local population. (1) Today, we remain true to that, and all our graduates practise in the public system, making this 'for Singaporeans, by Singaporeans'.

In our history of 113 years, the medical school has made many contributions to Singapore and its history mirrors that of modern Singapore. What some alumni may not know is that we now have a department of nursing, the Alice Lee Centre for Nursing Studies (ALCNS), which produces graduate nurses. This year, the intake of the nursing cohort exceeded that of the medical intake for the first time. That is proportionate to the workforce and entirely appropriate, as we need more nurses than doctors. The ALCNS will become its own school once it is ready, which will be quite soon.

Singapore medicine has come a long way. We have the second lowest infant mortality rate in the world. (2) The average Singaporean enjoys one of the highest life expectancies at birth. In the year 2000, the World Health Organization ranked Singapore among its 191 member states as the sixth most efficient healthcare system. ${ }^{(3)}$ In 2014, Bloomberg ranked Singapore healthcare system as the most efficient among 51 countries. ${ }^{(4)}$ In the United Nations' Sustainable Development Goals (SDGs), Singapore ranked No. 1 in terms of progress towards the SDGs, which include health, well-being and addressing poverty. ${ }^{(5)}$

\section{CHALLENGES TO OUR HEALTHCARE SYSTEM}

Collectively, the Singapore health system has done well. But we face many challenges in the future. Firstly, the population is ageing very rapidly. Secondly, the workforce will contract, including the health professional workforce of nurses and doctors. By 2030, the workforce will start contracting. ${ }^{(6)} \mathrm{We}$ have to prepare and make the necessary adjustments before that. Thirdly, healthcare costs have been escalating, and this is a burden on consumers, patients and the population. We must do what we can to control healthcare costs. The fourth problem is fragmentation. The system provides very deep and expert care on many levels, but there is room to improve in terms of coordination and holism. We could coordinate care better for the average patient, whose care can be better managed by a single family physician providing holistic care, rather than by multiple specialists.

These are some of the challenges. The Ministry of Health is addressing them. You have heard of the three shifts in healthcare: moving to health as opposed to healthcare; to community as opposed to treatment in hospitals; and to value, reflecting that we should always aim to give the best value to the population. The transformation of healthcare has already begun in terms of providing more community care and improving prevention.

\section{PREPARING FOR THE FUTURE}

Niels Bohr said, "Prediction is very difficult, especially about the future." But really, what we are trying to do is prepare for the future. Ultimately, we are training the next generation of doctors to address the healthcare needs of the population.

We could start by looking at what the pressing issues of the future are. We can then prepare our doctors for these challenges as well as we can. There are too many to go through, so I have selected five trends: technology; changes in practice patterns; changes in the role of the doctor; science and innovation in medicine; and preparing doctors to be future-ready doctors.

\section{TECHNOLOGY}

Technology is overwhelming today. How do we cope with technology, how do we utilise it to become efficient, more productive, but not lose the heart of clinical care and the doctorpatient relationship? 


\section{Technology transforming medical education}

We can think of technology in two facets. Firstly, how technology supports or assists medical education, and second, how it supports and changes the face of healthcare itself. Here are four ways in which technology is already transforming medical education: it serves as a means to engage and interact with students; broadens networks and allows collaborative work; enables personalised medical education; and widens access and acts as a leveller. All three schools in Singapore are already utilising these four ways as means to engage and interact with students.

Online learning and collaborative learning allow the student to personalise medical education. Using online learning, you can pick your own scheme of learning. You can choose your own materials. In the past, when we were sitting in a medical lecture hall, it was too slow or too fast for some of us. Using online learning, you can learn at your own pace. It widens access: less privileged populations can access online learning. They can tap on other medical schools for material.

Simulation training is another form of technology, which students use at the NUS Centre for Healthcare Simulation. Simulation training gives the students practical, hands-on experience in a safe and realistic manner. Students also have access to virtual anatomy, as a new, additional mode of learning anatomy. The students say that this improves on the old way of learning anatomy by giving them a very vivid threedimensional perspective. Previously, we studied anatomy from two-dimensional atlases and brain dissections. But in dissections, sometimes we damage or disturb certain structures through our lack of physical dexterity. In the virtual world, you can peel away layers and appreciate the three-dimension structure.

In summary, educational technology offers all of these modalities, including digital resources, and the ability to give simulation training. It is an online portal that is on all the time and can be accessed 24 hours a day, whenever you want to revise information.

\section{Technology supporting healthcare}

We turn now to the other facet of technology - how it supports healthcare. This includes scans, robotic surgery, electronic medical records (EHR) and genomic medicine. Students are already learning how to use EHR. In the wards, they access the hospital's EHR under supervision.

I also want to talk about artificial intelligence (AI). People are asking if Al robots will take away the job of doctors and how they will change medicine. In the National University Health System, we have performed some early studies using Al and natural language processing. The early results suggest that $\mathrm{Al}$ is not better than an experienced doctor, but if we use Al intelligently, it actually augments the diagnostic ability of the doctor. Al supplies checklists and prompts that may be useful for a junior doctor.

Another example of $\mathrm{Al}$ in medical practice would be in image diagnosis, for example, mammography reading. The present protocol is that mammograms are read by two radiologists. Trials have now been done in which the second radiologist is replaced by Al. Mammograms would be read by the human radiologist and Al. The human radiologist would then look at the Al's report and consider whether he wants to modify his own report. It is ultimately up to the radiologist. Rather than Al, we should think about the term 'augmented intelligence'. Effective use of information technology augments rather than replaces human intelligence.

I want to conclude this segment by saying that technology will never replace the human touch. Technology may facilitate care, but it cannot replace the heart of medical practice: the doctorpatient relationship and our ethos of care. More than ever, our patients want our care, compassion and reassurance, and this cannot be given by machines.

\section{CHANGES IN PRACTICE PATTERNS}

I shall now move on to the second trend: changing practice methods. In the old world, doctors practised as individuals; now, we work in teams. Previously, practice was based on expert opinion; it is now based on systematic reviews and evidence. In the old world, the doctor-patient relationship was paternalistic; now, it is based more on partnership. In the old world, most of what doctors needed to know was kept in their heads, but now with information technology, that is not necessary. A final, sobering observation is that we used to work many long hours, but the future generation may emphasise more work-life balance.

The doctor-patient relationship will change. Doctors are still respected and held in high esteem. But here are a few ways in which the relationship may change. First, the democratisation of medical knowledge. In the clinic, you have 10-20 minutes to see your patient. Under time pressure, you have to deal with a mass of information. The patient may bring in tests and reports. There is a lot of information to go through and you are seeing it for the first time. Doctors also have to walk a very fine line in terms of patient expectations. Modern medicine brings many capabilities, but of course there are limits, and we have to walk a fine balance between being realistic and destroying their hope. Finally, patients are looking for someone to interpret the information through the lens of experience and help them to distil and focus it.

There is a widespread opinion that we need more generalists. What is a generalist? The generalist approach is a philosophy of care that is distinguished by: a commitment to the breadth of practice within each discipline; collaboration with the larger healthcare team; and holistic management of the patient, including psychosocial factors, in order to respond to patient and community needs. ${ }^{(7)}$ Some generalist specialties include family medicine, internal general medicine, and geriatric and paediatric care.

Even within specialties, we can have a generalist mindset. For example, a neurologist can look after general medicine issues of diabetes mellitus and hypertension in a patient rather than referring the patient to another specialist. We need to exercise a broader practice set than has been the norm in recent years. 


\section{CHANGING ROLE OF THE DOCTOR}

When we were trained as doctors, we were prepared with the requisite skills, attitudes and knowledge to treat patients. We were prepared for roles as doctors looking after individual patients. Currently, the Ministry of Health is seeking to transform the healthcare system, and surely doctors have a role in that. We have to lead the transformation of the healthcare system across the whole country, and that is something we were not trained for. We will need to prepare our present students and future doctors better. We will be joined by other healthcare professionals, but it is likely that medical doctors will have to take a major share of the leadership in this. This will be a change in the role of doctors, beyond just treating and serving individual patients, to planning and transforming the healthcare system. The role of the doctor is changing from just providing good care to leading the transformation to better care. There is a lot of literature on this. There is a need for medical schools to train future physicians to continually improve the delivery of healthcare in order to achieve the full potential benefit for the health and well-being of the population. Existing textbooks cover current practice and do not address what optimal care might comprise in the future. We cannot take the current textbooks too literally at present. In the future, we have to write our own textbooks that are more suited to our context and update them with the needs of modern practice and what the patients need.

\section{MEDICINE, SCIENCE AND INNOVATION}

The fourth trend I am picking up has to do with medicine, science and innovation. Harnessing science to improve health is the unique opportunity of our time. We stand at a unique point in time, with technology and science capabilities that are unprecedented in history, at a time when Singapore healthcare is in a good position to take advantage of it. The transformation of care has begun and new innovations are needed. We have the opportunity to use these innovations to further improve the health of people and to transform our health system. The desired outcomes are: better population health; improved prevention; stronger community health services; and healthy ageing.

I will give some examples taken from clinical research in Singapore. The first example is the treatment of childhood acute lymphoblastic leukaemia (ALL), which is the commonest childhood cancer, led by Prof Allen Yeoh of the NUS Department of Paediatrics. Prof Yeoh has shown that ALL is caused by different types of genetic aberrations that influence the outcome of chemotherapy treatment. By genotyping the specific ALL variant in the individual child, the doctor can then select the treatment that would be appropriate for that genotype. A milder variant that responds well to treatment can be treated with a less intensive regime with fewer side effects. More aggressive subtypes such as those with deletion of the Ikaros gene will benefit from a more intensive chemotherapy regime. ${ }^{(8)}$

A second example is dengue fever, which is endemic in Southeast Asia. There are new vaccines for dengue, but these are not effective against all the genotypes. Prof Paul MacAry from the NUS Department of Microbiology has come up with a neutralising antibody for dengue. ${ }^{(9)}$ This could be a life-saving therapy for very severe cases of dengue haemorrhagic fever with shock. Administering this human neutralising antibody neutralises the dengue toxin and rescues the patient from the crisis of dengue shock.

A third example is from developmental research, by Prof Chong Yap Seng's translational research programme GUSTO (Growing Up in Singapore Towards Healthy Outcomes). ${ }^{(10,11)}$ Prof Chong followed up a cohort of babies and mothers who are now 10-12 years old, and made recommendations for practice policy and screening of maternal diabetes mellitus in Singapore. These guidelines have now been adopted nationally after the work by KK Women's and Children's Hospital and NUS. This shows that clinical research in Singapore has made an impact on the adoption of practical clinical guidelines for our population.

The final example comes from immunotherapy, CAR-T (chimeric antigen receptor T-cells) treatment, ${ }^{(12,13)}$ which is about activating T-cells to attack cancer. They are chimeric because the receptor molecule binds to the antigen and activates the T-cell to attack the cancer. Prof Dario Campana is a leading expert and a National Medical Research Council STaR (Singapore Translational Research Investigator Award) Investigator at NUS.

The formation of the Chapter of Clinician Scientists, Academy of Medicine, reflects the development of clinician scientists in the local healthcare system. The chapter aims to support training and development of clinician scientists and junior clinician scientists, and to promote academic medicine in Singapore. Formed in 2012, the chapter has now been upgraded to College status, with over 70 members, attesting to the growth of the clinician scientist community in Singapore.

\section{THE FUTURE-READY DOCTOR}

The final trend I will address is the future-ready doctor. What is 'future-ready'? The future-ready doctor has been described as having the following qualities: independent and critical thinker; able to adapt to new knowledge, interventions, therapeutics, and changing patterns of illness and health systems; ${ }^{(14)}$ the six ACGME (Accreditation Council for Graduate Medical Education) core competencies; ${ }^{(15)}$ personal excellence in clinical and humanistic skills; mastery of scientific foundations of health system performance; ${ }^{(16)}$ looking after an older population; ${ }^{(17)}$ quality improvement; ${ }^{(18)}$ a team approach to care; cost-effective care; ${ }^{(19)}$ health advisor and wellness coach; ${ }^{(20)}$ and complexity of healthcare. ${ }^{(21)}$ Healthcare today is more complicated, with more things to consider and more options, and management of the individual patient is also more complicated. How do we train this skill of dealing with complexity?

What are we doing today to prepare for these future qualities needed in doctors? We need the heart (values), head (knowledge), mind (qualities) and hands (skills). Beyond the traditional notion of a doctor, with the qualities of the heart, care, compassion and empathy; qualities of the head such as medical knowledge, lifelong learning and technology; and skillsets, competencies, safety and communication, I have added a new category of qualities of the mind, referring to the mindset of working in 
teams, being leaders, thinkers, innovators and improvers who can manage complexity. All these are qualities we will need in doctors in future. How do we prepare students, the curriculum and the faculty?

\section{Develop outcome-focused curriculums with clearly defined end-product capabilities ${ }^{(22)}$}

In our curriculum, we are focusing on future healthcare needs: healthy ageing, ageing population, prevention and promotion of health, evidence-based care and cost-effective care. Those will be outcomes we want to train for.

\section{Preparing graduates to have a sense of responsibility to the community ${ }^{(23)}$}

I spoke earlier about inculcating a sense of public mission in all our graduates and the ethos of service, which is the heart and root of medicine. NUS Medicine uses five strategies in the curriculum to prepare future-ready doctors: values-driven action; mastery-based learning; community-centric practice; outcome-focused evaluation; and aspirational vision and mission. Values drive all that we do. Mastery refers to acquiring generalist and specialist skills. Community-based practice refers to community services, family medicine, care and recuperation and regional health services. Outcome-focused refers to looking for better patient and health outcomes, looking at data and taking an evidence-based approach. Aspirational vision refers to our aspiration for better population health and improving our healthcare system.

\section{Emphasising values}

We believe that the right values will lead to the right choices and actions, ownership and accountability, patient-centred care, value consciousness and cost-effective care, and prevent many moral hazards.

\section{Selection of medical students}

Everything starts with the selection of medical students. More than eight years ago, we revamped the admissions system at NUS Medicine. It is now based on the MMI (multiple mini interview) format with focused skills assessment and a situational judgement test. Once you get to the interview stage, we do not consider results anymore but rather look for communication skills, behaviour that shows empathy and compassion, judgement of situations and appropriate actions.

\section{Values-driven action}

We have integrated the values of empathy and serving the community into the curriculum in many different formats: longitudinal patient; serving the community; houses; role models; team training; silent mentors; and caregiver training.

\section{Community service and service learning}

It is difficult to teach values and ethics in the classroom. We prefer to inculcate these by service learning and community service. Three examples of programmes are the Public Health Service, Neighbourhood Health Service and Tri-Generational HomeCare
@ North West. Public Health Service has been running for over 14 years. We bring medical students, nursing students, social work students and dental students to serve a community of several hundred over a weekend, providing health screening and health education. This allows students to practise their skills and serve the community as a way of exercising care and compassion.

\section{Commitment to professionalism}

All medical students at NUS Medicine are now asked to sign a two-page Commitment to Professionalism undertaking, a commitment to maintain and develop the professional attributes of honesty, integrity, responsibility, participation, respect, sensitivity, compassion and empathy, in the first week of medical school.

\section{Team approach to care}

Many physicians are increasingly functioning as part of a patient care team. Often, a physician may act as a leader of a patient care team whose other members might include nurse practitioners, physician assistants, medical educators and health aids. We train medical students through team training, developing their skills in communications, teamwork, patient safety and professionalism in the Centre for Healthcare Simulation. They practise working in teams with a strong focus on the need for inter-professional education, referring to the common foundation that all healthcare workers need, whether you are nurses, doctors or allied health professionals: patient safety; responsibility; and respect for persons. The CREATIVE programme stands for 'creating real-life experiences and teamwork in a virtual environment'. The students roleplay the roles of nurse, doctor, social worker and allied health professionals to solve patient problems, so that they get experience doing so. This gives students the opportunity to engage in multidisciplinary bedside rounds and discharge care planning.

\section{Promoting inquiry and innovation}

In the NUS Medical Grand Challenge, students are given one year to work in teams to solve unmet healthcare needs. Medical students team up with students from any two other faculties, forming a team of at least three, to solve medical or healthcare challenges. The 'eVand' is a lightweight leg sleeve, a project that won the Change Maker Prize this year. As you walk and move, it sends neuroelectrical impulses to the leg to contract the muscles and prevent deep vein thrombosis.

\section{Mastery}

COMPASS is our new curriculum with over 200 entrustable professional activities (EPAs). Students receive a document that describes the skills you need to become a doctor at the end of five years in the form of 200 EPAs. What are EPAs? Being able to take a history or identify a differential diagnosis of pyrexia of unknown origin would be an EPA. Master 200 EPAs and they have the foundation blocks to be a competent doctor. Students receive this document and tick off all the EPAs until they complete everything in Year 5.

We also need to teach students how to learn, because they need to be lifelong learners. After they graduate from medical 
school, there are still new skills to acquire that did not exist when they graduated. We practise this in terms of collaborative learning cases. They learn practical skills through experiential learning. We also use standardised patient material and give feedback to the students. Students now also get global medicine electives. Each year, about $90 \%$ of them join institutions in different countries, doing electives where they can see a different type of medicine and learn that there is not just Singapore medicine but a whole spectrum around the world.

Something that we did not do in your time but that we introduced from 2009 is the Graduate Exit Questionnaire (GEQ). After their final year exams, graduates are asked to complete this questionnaire on how satisfied they are with the quality of medical education across the five years. During the period of the old curriculum (2009-2012), the GEQ satisfaction rate was about $63 \%$, going up to $78 \%$ in 2013 . Since the implementation of the new curriculum from 2014, the GEQ satisfaction rate has been between $89 \%-95 \%$. This year's was $94 \%$. I think the increase in the satisfaction rate of the medical graduates attests to the quality of medical education and teaching they receive at all our teaching units all over Singapore. Thank you to all the teachers for the excellent teaching for our students; it is certainly much appreciated.

\section{CONCLUSION}

To summarise, tomorrow's doctors will need new skills to be future-ready. In my view, the following are important issues to consider in how we prepare and train medical students.

1. Leadership skills and to be a team player.

2. Soft skills will be more important than knowledge. In the electronic era, you can 'pull' knowledge and information, but soft skills such as bedside manner, how you communicate with patients and reassure them, how you answer their questions and your attitude towards them, must be intrinsic in the doctor.

3. We can retrieve a lot of things now and reduce some of the burden on learning and memory, because non-emergency knowledge can be 'pulled' when needed.

4. An underlying sense of responsibility towards our community and the patients we serve, strong sense of values and ethos.

5. Generalist skills: family medicine, general medicine, geriatrics, health promotion and prevention.

6. Skills for lifelong learning.

7. We need to deliver cost-effective care, and understand our health system, its costs, finances and economics.

8. Skills for quality improvement and innovation. We need to be comfortable with technology, data and analytics, in how to use medical data in our IT systems to improve our care, and for digital medicine.

9. Doctors will need a high degree of adaptability given the complexity and change in systems.

10. Continued trust and support from the community. We must always maintain trust, because that underlies everything we do and our ability to serve our patients.
Do these new skills mean that we need more paths in education? Education is a continuous sequence from undergraduate to postgraduate/specialty/family medicine training and continuing education. To learn how to acquire leadership skills, bring about more cost-effective care, change the healthcare system and transform care, will we not need other skills that are not currently provided in the curriculum? Research and innovation training is provided via the $\mathrm{MCl}$ (master of clinical investigation) or $\mathrm{PhD}$ in research. We need policy training, population health training (e.g. master of public health), management and financial training to run a healthcare system. Many chief executives of hospitals are doctors and possess an extra set of skills. If you have these skills, you will be more versatile and more valuable to your patients and to the community.

Here are some suggestions for the junior doctors and students. Possessing the right values, especially empathy and being there for your patients, will keep you in universal demand by both patients and employers. Develop mastery in your knowledge and skills, including being able to work with machines, data and Al. Align with the healthcare needs of Singapore. Be a lifelong learner, continue to acquire new skills. Travel in teams, because we have to go far together. Be adaptable and flexible, and not be disappointed when things do not work out. We must inculcate the ability to accept failure. It is okay to fail; pick yourself up and get on with the job.

What will protect our role as doctors in the future is our aspiration to make things better, to improve the system, because machines will not do this; to manage complexity, the grey and ambiguous areas; leadership skills to lead a team of junior doctors, nurses and health workers; team skills to organise multidisciplinary care; understanding of health systems and health finance; informatics and analytical skills; and being part of the team, with common goals, bringing valuable skills. With these added skills, you are also more valuable in the eyes of the employers.

Our alumni and senior doctors are important as role models and mentors to our junior doctors and students. Over 3,000 senior doctors in the public and private sector are appointed as clinical teachers, as interviewers for selection at admissions and as mentors. Our 10,000 alumni are a human bridge between the school's past and our future, and I hope that in the future, many more alumni will come back to play active roles in our education and academic mission, and help us train the next generation of doctors. I thank Prof Wong Tien Yin for chairing the SMA Charity Fund. The Charity Fund has disbursed over 300 bursaries, more than SGD 1.5 million since 2007 to medical students across all three schools. Please join us in ensuring that financial need is not a barrier to students with the ability and qualifications to study medicine and contribute to the community. Your support will be greatly appreciated.

The future is bright and exciting, but it will not be easy. The challenges facing our healthcare system are great, and the key to overcoming these challenges lies in medical education and how we prepare our next generation of doctors. 


\section{REFERENCES}

1. Lee EJD, ed. To Sail Uncharted Seas: Commemorating 100 Years o Medical Education (1905-2005). 1st ed. Singapore: National University of Singapore, 2005.

2. Department of Statistics, Singapore. Infant Mortality in Singapore. Statistics Singapore Newsletter September 2009. Available at: https://www.singstat. gov.sg/-/media/files/publications/population/ssnsep09-pg18-19.pdf. Accessed November 1, 2018.

3. World Health Organization. The World Health Report 2000 - Health Systems Improving Performance. Available at: https://www.who.int/whr/2000/en/ whr00 en.pdf?ua=1. Accessed November 1, 2018.

4. Bloomberg.com. "Where Do You Get the Most for Your Health Care Dollar?" Available at: https://www.bloomberg.com/graphics/infographics/most-efficienthealth-care-around-the-world.html. Accessed November 1, 2018.

5. GBD 2016 SDG Collaborators. Measuring progress and projecting attainmen on the basis of past trends of the health-related Sustainable Development Goals in 188 countries: an analysis from the Global Burden of Disease Study 2016. Lancet 2017; 390:1423-59.

6. Ministry of Health, Singapore. I Feel Young in My Singapore!: Action Plan for Successful Ageing 2016. Available at: https://www.moh.gov.sg/docs/ librariesprovider3/action-plan/action-plan.pdf. Accessed November 1, 2018.

7. Royal College of Physicians and Surgeons of Canada. Report of the Generalism and Generalist Task Force. July 2013. Available at: http://www.royalcollege.ca/ rcsite/documents/educational-strategy-accreditation/ccc-task-force-report-july2013-e.pdf. Accessed November 1, 2018.

8. Yeoh AEJ, Lu Y, Chin WHN, et al. Intensifying treatment of childhood B-lymphoblastic leukemia With IKZF1 deletion reduces relapse and improve overall survival: results of Malaysia-Singapore ALL 2010 study. J Clin Oncol 2018; 36:2726-35

9. Rivino L, Kumaran EA, Thein TL, et al. Virus-specific T lymphocytes home to the skin during natural dengue infection. Sci Transl Med 2015; 7:278ra35.

10. De Seymour J, Chia A, Colega M, et al. Maternal dietary patterns and gestational diabetes mellitus in a multi-ethnic Asian cohort: the GUSTO study. Nutrients
2016; 8:E574.

11. Cai S, Qiu A, Broekman BF, et al; GUSTO study group. The influence of gestational diabetes on neurodevelopment of children in the first two years of life: a prospective study. PLoS One 2016; $11: \mathrm{e} 0162113$.

12. Kudo K, Imai C, Lorenzini $P$, et al. T lymphocytes expressing a CD16 signaling receptor exert antibody-dependent cancer cell killing. Cancer Res 2014; 74:93103

13. Imamura M, Shook D, Kamiya $T$, et al. Autonomous growth and increased cytotoxicity of natural killer cells expressing membrane-bound interleukin-15. Blood 2014; 124:1081-8.

14. The Association of Faculties of Medicine of Canada. The Future of Medical Education in Canada (FMEC): A Collective Vision for MD Education. Available at: http://cou.on.ca/wp-content/uploads/2010/01/COU-Future-of-MedicalEducation-in-Canada-A-Collective-Vision.pdf. Accessed November 1, 2018.

15. Accreditation Council for Graduate Medical Education. 1999 Annual Report. Available at: https://www.acgme.org/Portals/0/PDFs/an_1999AnnRep.pdf. Accessed November 1, 2018.

16. Berwick DM, Finkelstein JA. Preparing medical students for the continual improvement of health and health care: Abraham Flexner and the new "public interest". Acad Med 2010; 85(9 Suppl):S56-65.

17. Pershing S, Fuchs VR. Restructuring medical education to meet current and future health care needs. Acad Med 2013; 88:1798-801.

18. Wong BM, Levinson W, Shojania KG. Quality improvement in medical education: current state and future directions. Med Educ 2012; 46:107-19.

19. Pershing $S$, Fuchs VR. Restructuring medical education to meet current and future health care needs. Acad Med 2013; 88:1798-801.

20. Morrison I. The future of physician's time. Ann Intern Med 2000; 132:80-4.

21. Rouse WB, Serban N. Un=derstanding and Managing the Complexity of Healthcare. 1st ed. Cambridge: MIT Press, 2014

22. Wilkinson D. The future of medical education: all about being connected. Ochsner J 2012; 12:300-1.

23. Woollard RF. Caring for a common future: medical schools' social accountability. Med Educ 2006; 40:301-13.

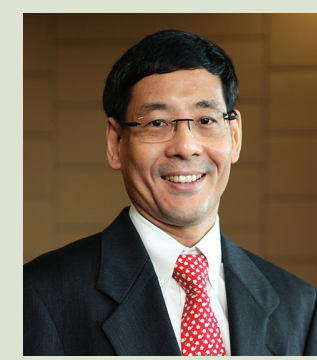

\section{About the Lecturer}

A/Prof Yeoh Khay-Guan is concurrently Dean of Yong Loo Lin School of Medicine, National University of Singapore, and Deputy Chief Executive, National University Health System. He practises as a Senior Consultant at the Department of Gastroenterology and Hepatology, National University Hospital. His research interest is in enhancing the early detection of gastric and colorectal cancers. He is the Lead Principal Investigator of the Singapore Gastric Cancer Consortium, a national flagship research group, which aims to improve the outcomes for gastric cancer in Singapore. He also chairs the National Colorectal Cancer Screening Committee of the Health Promotion Board, Ministry of Health, which recommends guidelines for the nationa colorectal screening programme in Singapore.

The 2018 SMA Lecture was delivered on 10 November 2018 at Novotel Singapore on Stevens. The citation of A/Prof Yeoh Khay Guan was delivered by A/Prof Chin Jing Jih, Deputy Chairman Medical Board, Tan Tock Seng Hospital and former SMA President (2012-2015). A copy of the citation was published in the December 2018 issue of the SMA News, and the video of the lecture is available at https://wWw.sma.org.sg/aboutus/index.aspx?ID=136 (SMA Member login required). 